\title{
Robust beamforming and cooperative jamming for secure transmission in DF relay systems
}

\author{
Bin Li and Zesong Fei*
}

\begin{abstract}
In this paper, we study robust joint beamforming and cooperative jamming (CJ) in a secure decode-and-forward (DF) relay system in the presence of multiple eavesdroppers, in which a multi-antenna DF relay employs transmit beamforming to help the source deliver information to the destination and simultaneously generates Gaussian artificial noise to confuse these eavesdroppers. We assume that all the channel state information (CSI) is imperfectly known at the relay subject to norm-bounded CSI errors. Our objective is to maximize the achievable secrecy rate at the destination by jointly optimizing the transmit beamforming vector of information signals and the covariance matrix of jamming signals at the relay subject to its total transmit power constraint. Although this problem is non-convex and in general difficult to be solved, we obtain its optimal solution via the technique of semi-definite relaxation (SDR) together with a one-dimensional (1-D) search. Specifically, we prove that the SDR is tight for the problem of our interest. Finally, numerical results are provided to validate our proposed scheme.
\end{abstract}

Keywords: Physical layer security, Decode-and-forward (DF) relay, Cooperative jamming (CJ), Robust beamforming, Semi-definite relaxation (SDR)

\section{Introduction}

Secure wireless transmissions have drawn significant attention due to the broadcast nature of radio propagation and the inherent openness of the transmission medium. In addition to the traditional encryption techniques, physical layer security has been recognized as a prominent technique to realize secure communication by exploiting wireless channel fading (see, e.g., [1-3]). Recently, the introduction of relay into physical layer security has attracted a lot of research interest as a promising solution to improve transmission security through cooperative diversity $[4,5]$. Similar to the traditional relay systems, amplify-and-forward (AF) [6] and decode-and-forward (DF) [7] are two commonly used schemes in secure relay systems.

To further improve physical layer security, artificial noise (AN) or cooperative jamming (CJ) approach has been introduced in the secrecy system by embedding

*Correspondence: feizesong@bit.edu.cn

School of Information and Electronics, Beijing Institute of Technology, Beijing 100081, China noise or jamming signal in the transmitted signal to confuse eavesdroppers [8-11]. In addition to helping relay the desirable information signals from source nodes to destination nodes, trusted relays can also implement $\mathrm{CJ}$ $[12,13]$ to confuse the potential eavesdroppers by simultaneously sending jamming signals together with the information signals. Furthermore, due to the success of multi-antenna techniques in wireless communications, employing multiple antennas in the secure relay systems has also been investigated in the literature recently [14-16], in which the multi-antenna nodes can adjust the directions of their transmitted information and jamming signals so as to further improve the secure performance.

Recently, there are some works [17-19] in the literature investigating joint relay beamforming and CJ design to maximize the achievable secrecy rate in relay systems. Specifically, [17] studied secure beamforming and $\mathrm{CJ}$ design in an AF relay system with multiple distributed relays and multiple eavesdroppers each with a single antenna, in which the secrecy rate was maximized subject to both total and individual power constraints at the

\section{Springer}


relay nodes. Ding et al. [18] considered joint beamforming and CJ scheme in an AF-based multi-antenna relay system with an eavesdropper, in which interference alignment was used to ensure that jamming signals from the source and the relay can be aligned together at the destination. The most recent work [19] addressed DF-based multi-antenna relay beamforming and $\mathrm{CJ}$ design in the presence of multiple eavesdroppers. In [17-19], the global channel state information (CSI) is assumed to be perfectly known at both the source and the relay.

However, the practical implementation of beamforming and CJ design at the multi-antenna relay in secure wireless communications critically relies on the CSI availability from the source/relay to the destination/eavesdroppers at the transmitter [20-23]. This is difficult to be obtained perfectly in practice due to channel quantization and feedback errors. As a result, it is essential to investigate the robust relay transmission design under imperfect CSI. Robust transmit designs for multiple-input singleoutput (MISO) wiretap channels under both individual and global power constraints for the source and the helper were presented in [21], where transmit covariance matrices at the source and the helper were jointly optimized to maximize the secrecy rate. Zhao et al. [22] investigated robust beamforming design of sum secrecy rate optimization under sum power constraint for multiple usereavesdropper pairs, and a low complexity zero-forcing (ZF) beamformers is developed. Ni et al. [23] and Fei et al. [24] discussed robust coordinated beamforming for multiuser interference channel secure communications under imperfect CSI. There have been no consideration of the relay system in [21-24]. It is worth noting that there has been a handful of works [25-27] investigating the robust relay beamforming design. Specifically, [25] investigated the AF-based relay beamforming scheme in the presence of multiple eavesdroppers to maximize the secrecy rate. The imperfect CSI of relay-destination and relayeavesdroppers links was modeled using a norm-bounded CSI error model. Zhang et al. [26] designed robust joint optimization in bidirectional multi-user multi-relay MIMO systems, in which authors mainly concentrated on the sum mean square error (MSE) criterion as well as maximum user's MSE. However, the two aforementioned works did not consider jamming signals generated by relays to confuse eavesdroppers. Wang and Wang [6] has considered an AF relaying system with the multiple distributed relays each with a single antenna. In [27], the relaying robust beamforming for device-to-device communication with channel uncertainty was considered. To the best of our knowledge, the DF-based robust joint beamforming and $\mathrm{CJ}$ design for maximizing secrecy rate in relay systems with imperfect CSI is much less known.

In this paper, we focus on the robust joint beamforming and CJ design for a DF-based relay system in the presence of multiple eavesdroppers, in which a single-antenna source sends confidential information to a single-antenna destination with the assistance of a multi-antenna relay, and a jamming signal is embedded in the transmitted signal of relay to improve secure communication. The contributions of this paper are twofold:

1) The relay operates in a half-duplex mode, accordingly the transmission is divided into two time slots. In the first time slot, the source transmits signals to the destination and the relay, and in the second time slot, the relay forwards the re-encoded signals (a jamming signal is embedded in the transmitted signal of relay) to the destination. It is presumed that the wireless channels from the source/relay to the destination/eavesdroppers are subject to norm-bounded CSI errors.

2) By jointly optimizing the transmit beamforming vector of information signals and the covariance matrix of jamming signals at the relay to maximize the achievable secrecy rate at the destination while guaranteeing the total transmit power constraint. Although this problem is non-convex and in general difficult to be solved, we obtain its optimal solution via the techniques of semi-definite relaxation (SDR) together with a one-dimensional (1-D) search. Furthermore, we prove that the tightness of SDR of channel uncertainty, which can guarantee the optimality of the solution in this case.

The rest of this paper is organized as follows. Section 2 introduces the system model and problem formulation. Section 3 proposes the optimal solution to the considered problem. Section 4 presents numerical results to validate the proposed scheme. Finally, Section 5 concludes this paper.

Notations: Throughout this paper, boldface lowercase and uppercase letters denote vectors and matrices, respectively. The transpose, conjugate transpose, rank, and trace of matrix $\mathbf{A}$ are denoted as $\mathbf{A}^{T}, \mathbf{A}^{H}, \operatorname{rank}(\mathbf{A})$, and $\operatorname{tr}(\mathbf{A})$, respectively. $\mathbf{A}^{*}$ denotes the conjugate of matrix $\mathbf{A}$, and $\mathbf{I}$ denotes the identity matrix. $\mathbf{A} \succeq 0$ means $\mathbf{A}$ is a positive semidefinite matrix. The distribution of a circularly symmetric complex Gaussian (CSCG) random vector with mean vector $\mathbf{x}$ and covariance matrix $\boldsymbol{\Sigma}$ is denoted by $\mathcal{C N}(\mathbf{x}, \Sigma)$; and $\sim$ stands for "distributed as."

\section{System model and problem formulation}

\subsection{System model}

We consider a DF relaying system, which consists of one source node (S), one relay node (R), one destination node (D), and $K>1$ passive eavesdroppers (Es), as shown in 
Fig. 1. Suppose that the relay is equipped with $N>1$ antennas, and each of the other nodes is equipped with a single antenna. Let $\mathcal{K}=\{1, \ldots, K\}$ denote the set of Es. The relay operates in a half-duplex mode, and accordingly, the information transmission from $\mathrm{S}$ to $\mathrm{D}$ with the assistance of $\mathrm{R}$ requires two time slots to complete. In the first time slot, $\mathrm{S}$ transmits signals to $\mathrm{R}$ and $\mathrm{D}$. After decoding the information, in the second time slot, $\mathrm{R}$ forwards the reencoded signals to $D$. To enhance physical layer security by preventing the $K$ Es from eavesdropping the confidential signal sent by $\mathrm{S}$ to $\mathrm{D}$, we consider that $\mathrm{R}$ employs the CJ-aided secure communication scheme. Specifically, R employs transmit beamforming for delivering the confidential information to $\mathrm{D}$ and at the same time generates Gaussian artificial noise to confuse the $K$ Es.

Throughout this paper, the $K$ Es are passive and the goal is to interpret the transmission information without trying to modify it. It is assumed that $\mathrm{S}$ and $\mathrm{R}$ can (partially) obtain the CSI associated with Es through, e.g., estimating channels based on Es' transmitted signals, while they do not know the location of Eve. As Es' location changes, the proposed beamforming design is able to adapt dynamically to ensure the secure communications of Alice based on the associated channels. Note that the location-based secure robust beamforming design is beyond the scope of this paper, while the interested readers can refer to [28] and the reference therein for more information. Let the channel coefficients over links $S \rightarrow D, S \rightarrow E_{k}, S \rightarrow R$, $R \rightarrow D$, and $R \rightarrow E_{k}(k \in \mathcal{K})$ be denoted by $h_{\mathrm{SD}}, h_{\mathrm{SE}_{k}}$, $\mathbf{h}_{\mathrm{SR}} \in \mathbb{C}^{N \times 1}, \mathbf{h}_{\mathrm{RD}} \in \mathbb{C}^{N \times 1}$, and $\mathbf{h}_{\mathrm{RE}_{k}} \in \mathbb{C}^{N \times 1}$, respectively. All channel coefficients are quasi-stationary with flat-fading Rayleigh distribution.
Then, by denoting the confidential signal sent by $\mathrm{S}$ as $x$, the received signals at $\mathrm{R}, \mathrm{D}$, and $\mathrm{E}_{k}$ in the first time slot are respectively denoted as

$$
\begin{aligned}
& \mathbf{y}_{R}=\sqrt{P_{S}} \mathbf{h}_{\mathrm{SR}}^{T} x+\mathbf{n}_{R} \\
& y_{1 D}=\sqrt{P_{s}} h_{\mathrm{SD}} x+n_{1 D} \\
& y_{1 E_{k}}=\sqrt{P_{s}} h_{\mathrm{SE}_{k}} x+n_{1 E_{k}}
\end{aligned}
$$

where $P_{S}$ is the transmit power at the $\mathrm{S} . \mathbf{n}_{R}, n_{1 D}$, and $n_{1 E_{k}}$ are the complex Gaussian noises at the R, D, and $\mathrm{E}_{k}$ with $\mathbf{n}_{R} \sim \mathcal{C N}\left(\mathbf{0}, \sigma^{2} \mathbf{I}\right), n_{1 D} \sim \mathcal{C N}\left(0, \sigma^{2}\right)$ and $n_{1 E_{k}} \sim$ $\mathcal{C N}\left(0, \sigma^{2}\right)$, respectively. For notational convenience, we assume that the noise power at R, D, and Es are identical and normalized to be unity (i.e., $\sigma^{2}=1$ ) without loss of generality.

Using (1), the information rate at $\mathrm{R}$ is given by

$$
R_{R}=\frac{1}{2} \log _{2}\left(1+P_{s}\left\|\mathbf{h}_{\mathrm{SR}}\right\|^{2}\right)
$$

where the factor $1 / 2$ accounts for the fact that the overall transmission is divided into two time slots.

In the second time slot, $\mathrm{R}$ successfully decodes the source signal and then forwards the re-encoded signal to $\mathrm{D}$ together with a jamming signal. Specifically, let the sent signal of $\mathrm{R}$ in the second time slot be denoted by $\tilde{\mathbf{x}}=\mathbf{w} x+\mathbf{z}$, where $\mathbf{w} \in \mathbb{C}^{N \times 1}$ denotes the transmit beamforming vector carrying the confidential information and $\mathbf{z} \in \mathbb{C}^{N \times 1}$ denotes the complex Gaussian jamming signal generated by $\mathrm{R}$, which is a CSCG random vector with zero

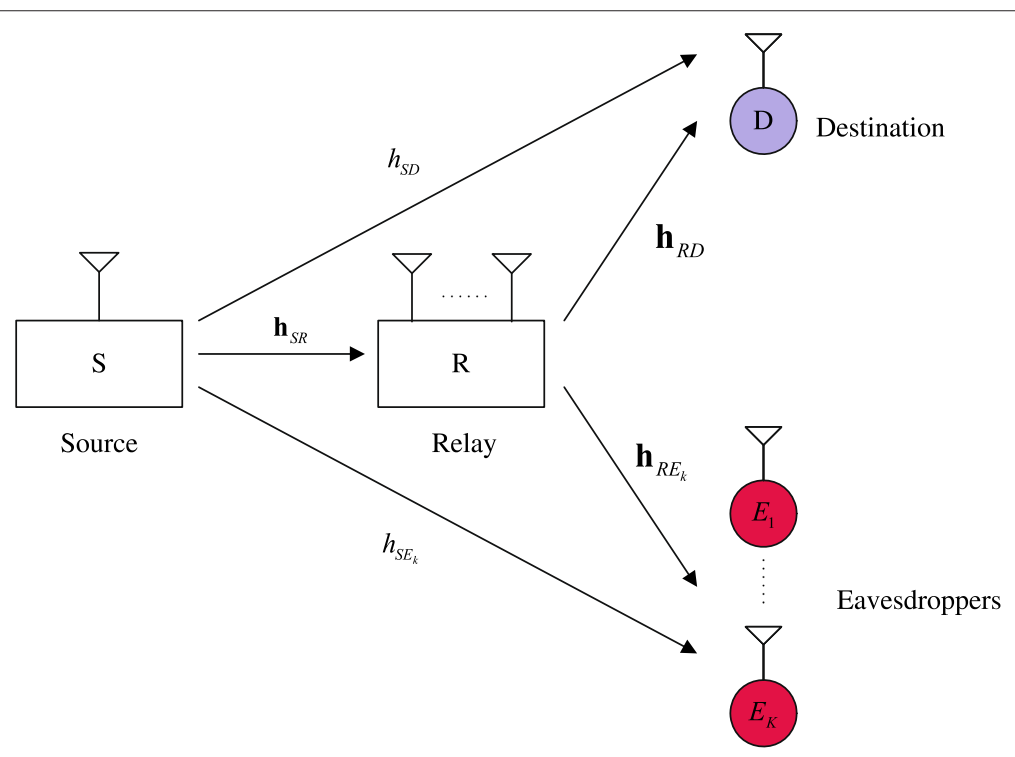

Fig. 1 System model of DF-based relay beamforming and CJ 
mean and covariance $\Omega \succeq \mathbf{0}$, i.e., $\mathbf{z} \sim \mathcal{C N}(\mathbf{0}, \Omega)$. Then, the received signals at $\mathrm{D}$ and $\mathrm{E}_{k}$ are respectively denoted as

$$
\begin{aligned}
& y_{2 D}=\mathbf{h}_{\mathrm{RD}}^{T} \mathbf{w} x+\mathbf{h}_{\mathrm{RD}}^{T} \mathbf{z}+n_{2 D} \\
& y_{2 E_{k}}=\mathbf{h}_{\mathrm{RE}_{k}}^{T} \mathbf{w} x+\mathbf{h}_{\mathrm{RE}_{k}}^{T} \mathbf{z}+n_{2 E_{k}}
\end{aligned}
$$

where $n_{2 D}$ and $n_{2 E_{k}}$ denote the complex Gaussian noises at $\mathrm{D}$ and $\mathrm{E}_{k}$ with $n_{2 D} \sim \mathcal{C N}\left(0, \sigma^{2}\right)$ and $n_{2 E_{k}} \sim \mathcal{C N}\left(0, \sigma^{2}\right)$, respectively.

Using maximum ratio combination (MRC) to combine (2) and (5) $[29,30]$, the information rate at $\mathrm{D}$ is given by

$$
R_{D}(\mathbf{w}, \Omega)=\frac{1}{2} \log _{2}\left(1+P_{s}\left|h_{\mathrm{SD}}\right|^{2}+\frac{\mathbf{h}_{\mathrm{RD}}^{T} \mathbf{w} \mathbf{w}^{H} \mathbf{h}_{\mathrm{RD}}^{*}}{1+\mathbf{h}_{\mathrm{RD}}^{T} \Omega \mathbf{h}_{\mathrm{RD}}^{*}}\right) .
$$

Similarly, using (3) and (6), the information rate at $\mathrm{E}_{k}$ by exploiting the MRC is given by

$$
R_{E_{k}}(\mathbf{w}, \Omega)=\frac{1}{2} \log _{2}\left(1+P_{s}\left|h_{\mathrm{SE}_{k}}\right|^{2}+\frac{\mathbf{h}_{\mathrm{RE}_{k}}^{T} \mathbf{w w}^{H} \mathbf{h}_{\mathrm{RE}_{k}}^{*}}{1+\mathbf{h}_{\mathrm{RE}_{k}}^{T} \Omega \mathbf{h}_{\mathrm{RE}_{k}}^{*}}\right), \forall k \in \mathcal{K} .
$$

For the secure MISO relay communication in the presence of multiple eavesdroppers, the achievable secrecy rate is given by [15]

$$
R=\min _{k \in \mathcal{K}} R_{D}(\mathbf{w}, \Omega)-R_{E_{k}}(\mathbf{w}, \Omega) .
$$

Following [12], to ensure the $\mathrm{R}$ correctly decode the source message, the rate at $\mathrm{R}$ should no less than the rate at $\mathrm{D}$, i.e.,

$$
R_{R} \geq R_{D}(\mathbf{w}, \Omega) .
$$

On the other hand, the total transmit power (signal power plus jamming signal power) at $\mathrm{R}$ is limited by the maximum allowable transmit power $P_{r}$, i.e.,

$$
\operatorname{tr}\left(\mathbf{w} \mathbf{w}^{H}\right)+\operatorname{tr}(\Omega) \leq P_{r}
$$

\subsection{Problem formulation}

In this paper, we consider imperfect CSI at the transmitter due to channel quantization and feedback errors. In other words, the CSI from S/R to D/Es is imperfectly known at $S$ and R. Here, the CSI uncertainties are modeled by worstcase model as in [21,31]. In this case, we consider that the estimated wireless channels over links $S \rightarrow D, S \rightarrow E_{k}$, $R \rightarrow D$, and $R \rightarrow E_{k}(k \in \mathcal{K})$ are denoted by $\tilde{h}_{\mathrm{SD}}, \tilde{h}_{\mathrm{SE}_{k}}$, $\tilde{\mathbf{h}}_{\mathrm{RD}}$, and $\tilde{\mathbf{h}}_{\mathrm{RE}_{k}}$, respectively. We assume that the channel uncertainty is bounded, and accordingly denote the regions of the exact wireless channels $h_{S D}, h_{S E_{k}}, \mathbf{h}_{R D}$, and
$\mathbf{h}_{R E_{k}}$ as $\mathcal{H}_{S D}, \mathcal{H}_{S E_{k}}, \mathcal{H}_{R D}$, and $\mathcal{H}_{R E_{k}}$, respectively, which are given by

$$
\begin{aligned}
& \mathcal{H}_{\mathrm{SD}}=\left\{\Delta h_{\mathrm{SD}}\left|\Delta h_{\mathrm{SD}}=h_{\mathrm{SD}}-\tilde{h}_{\mathrm{SD}},\right| \Delta h_{\mathrm{SD}} \mid \leq \delta_{\mathrm{SD}}\right\}, \\
& \mathcal{H}_{\mathrm{SE}_{k}}=\left\{\Delta h_{\mathrm{SE}_{k}}\left|\Delta h_{\mathrm{SE}_{k}}=h_{\mathrm{SE}_{k}}-\tilde{h}_{\mathrm{SE}_{k}},\right| \Delta h_{\mathrm{SE}_{k}} \mid \leq \delta \delta_{\mathrm{SE}_{k}}\right\}, \\
& \forall k \in \mathcal{K}
\end{aligned}
$$

$$
\mathcal{H}_{\mathrm{RD}}=\left\{\Delta \mathbf{h}_{\mathrm{RD}} \mid \Delta \mathbf{h}_{\mathrm{RD}}=\mathbf{h}_{\mathrm{RD}}-\tilde{\mathbf{h}}_{\mathrm{RD}},\left\|\Delta \mathbf{h}_{\mathrm{RD}}\right\| \leq \delta_{\mathrm{RD}}\right\}
$$

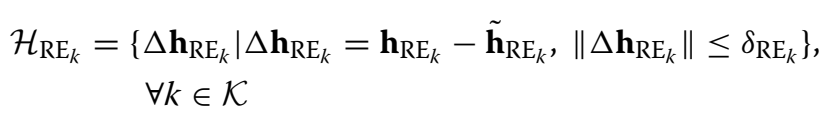

where $\Delta h_{\mathrm{SD}}, \Delta h_{\mathrm{SE}_{k}}, \Delta \mathbf{h}_{\mathrm{RD}}$, and $\Delta \mathbf{h}_{\mathrm{RE}_{k}}$ denote the channel uncertainties, respectively. And $\delta_{\mathrm{SD}}, \delta_{\mathrm{SE}_{k}}, \delta_{\mathrm{RD}}, \delta_{\mathrm{RE}_{k}}$ denote the error bounds corresponding uncertainty regions, respectively.

Under the channel uncertainty model, our objective is to maximize the achievable secrecy rate at $\mathrm{D}$ in (9) by jointly optimizing the beamforming vector $\mathbf{w}$ of the desirable signal and the transmit covariance $\Omega$ of the jamming signal at $\mathrm{R}$ subject to the information rate constraint in (10) and its total transmit power constraint at $\mathrm{R}$ in (11). As a result, the optimization problem is formulated as

$$
\max _{\substack{\mathbf{w}, \Omega \\ \Delta h_{S \mathrm{D}} \in \mathcal{H}_{\mathrm{SD}}, \Delta h_{S_{k}} \in \mathcal{H}_{S \mathrm{E}_{k}} \\ \Delta \mathbf{h}_{\mathrm{RD}} \in \mathcal{H}_{\mathrm{RD}}, \Delta \mathbf{h}_{\mathrm{RE}_{k}} \in \mathcal{H}_{\mathrm{RE}_{k}}}} \min _{k \in \mathcal{K}} R_{D}(\mathbf{w}, \Omega)-R_{E_{k}}(\mathbf{w}, \Omega)
$$

$$
\begin{array}{ll}
\text { s.t. } & R_{R} \geq R_{D}(\mathbf{w}, \Omega) \quad(16 \\
& \operatorname{tr}\left(\mathbf{w} \mathbf{w}^{H}\right)+\operatorname{tr}(\Omega) \leq P_{r}
\end{array}
$$

$$
\Omega \succeq \mathbf{0} .
$$

Problem (16) is challenging to be solved due to the non-convex objective function (16a) (the objective function is the difference of two logarithm functions) and the semi-infinite constraint (16b) (due to the norm-bounded CSI errors). We address this challenge in the following section.

\section{Optimal solution}

In this section, we employ the SDR technique to solve problem (16). Define $\mathbf{W}=\mathbf{w w}^{H}$ with $\mathbf{W} \succeq \mathbf{0}$ and $\operatorname{rank}(\mathbf{W}) \leq 1$. Then, by substituting $\mathbf{w} \mathbf{w}^{H}$ as $\mathbf{W}$ and introducing a slack variable $\alpha \geq 0$, problem (16) is equivalently expressed as 
$\begin{aligned} \max _{\mathbf{W}, \Omega, \alpha} \min _{\substack{\Delta h_{\mathrm{SD}} \in \mathcal{H} \\ \Delta \mathbf{h}_{\mathrm{RD}} \in \mathcal{H} \mathcal{H D}_{\mathrm{RD}}}} & \frac{1}{2} \log _{2}\left(1+P_{s}\left|h_{\mathrm{SD}}\right|^{2}+\frac{\mathbf{h}_{\mathrm{RD}}^{T} \mathbf{W h} \mathbf{h}_{\mathrm{RD}}^{*}}{1+\mathbf{h}_{\mathrm{RD}}^{T} \Omega \mathbf{h}_{\mathrm{RD}}^{*}}\right) \\ -\frac{1}{2} \log _{2} \alpha & \end{aligned}$

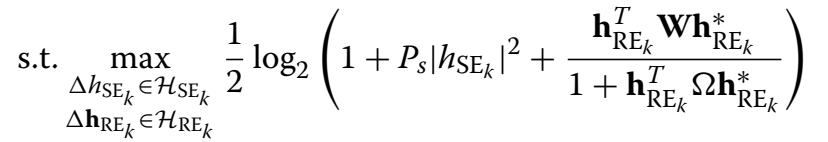

$\leq \frac{1}{2} \log _{2} \alpha, \forall k \in \mathcal{K}$ $\max _{\substack{\Delta h_{\mathrm{SD}} \in \mathcal{H}_{\mathrm{SD}} \\ \Delta \mathbf{h}_{\mathrm{RD}} \in \mathcal{H}_{\mathrm{RD}}}} \frac{1}{2} \log _{2}\left(1+P_{s}\left|h_{\mathrm{SD}}\right|^{2}+\frac{\mathbf{h}_{\mathrm{RD}}^{T} \mathbf{W} \mathbf{h}_{\mathrm{RD}}^{*}}{1+\mathbf{h}_{\mathrm{RD}}^{T} \Omega \mathbf{h}_{\mathrm{RD}}^{*}}\right)$

$$
\leq \frac{1}{2} \log _{2}\left(1+P_{S}\left\|\mathbf{h}_{\mathrm{SR}}\right\|^{2}\right),
$$

$\operatorname{tr}(\mathbf{W})+\operatorname{tr}(\Omega) \leq P_{r}$,

$\mathbf{W} \succeq \mathbf{0}, \Omega \succeq \mathbf{0}, \alpha \geq 0$,

$\operatorname{rank}(\mathbf{W}) \leq 1$.

Furthermore, introducing a slack variable $\gamma \geq 0$, problem (17) is reformulated as

$$
\begin{aligned}
& \max _{\mathbf{W}, \Omega, \alpha, \gamma} \gamma \\
& \text { s.t. } \min _{\substack{\Delta h_{\mathrm{SD}} \in \mathcal{H}_{\mathrm{SD}} \\
\Delta \mathbf{h}_{\mathrm{RD}} \in \mathcal{H}_{\mathrm{RD}}}} \frac{1+P_{s}\left|h_{\mathrm{SD}}\right|^{2}}{\alpha}+\frac{\mathbf{h}_{\mathrm{RD}}^{T} \mathbf{W} \mathbf{h}_{\mathrm{RD}}^{*}}{\alpha\left(1+\mathbf{h}_{\mathrm{RD}}^{T} \Omega \mathbf{h}_{\mathrm{RD}}^{*}\right)} \geq \gamma, \\
& \max _{\substack{\Delta h_{\mathrm{SE}_{k}} \in \mathcal{H}_{\mathrm{SE}_{k}} \\
\Delta \mathbf{h}_{\mathrm{RE}_{k}} \in \mathcal{H}_{\mathrm{RE}_{k}}}} 1+P_{s}\left|h_{\mathrm{SE}_{k}}\right|^{2}+\frac{\mathbf{h}_{\mathrm{RE}_{k}}^{T} \mathbf{W h}_{\mathrm{RE}_{k}}^{*}}{1+\mathbf{h}_{\mathrm{RE}_{k}}^{T} \Omega \mathbf{h}_{\mathrm{RE}_{k}}^{*}} \leq \alpha, \forall k \in \mathcal{K} \\
& \max _{\substack{\Delta h_{\mathrm{SD}} \in \mathcal{H}_{\mathrm{SD}} \\
\Delta \mathbf{h}_{\mathrm{RD}} \in \mathcal{H}_{\mathrm{RD}} \\
(17 \mathrm{~d})-(17 \mathrm{f}),}} P_{s}\left|h_{\mathrm{SD}}\right|^{2}+\frac{\mathbf{h}_{\mathrm{RD}}^{T} \mathbf{W h}_{\mathrm{RD}}^{*}}{1+\mathbf{h}_{\mathrm{RD}}^{T} \Omega \mathbf{h}_{\mathrm{RD}}^{*}} \leq P_{s}\left\|\mathbf{h}_{\mathrm{SR}}\right\|^{2},
\end{aligned}
$$

where function $\log _{2}(\cdot)$ is omitted since the logarithm is a monotonically increasing function that has no effect on the optimization problem.

To simplify problem (18e), we introduce three variables $a, b, c$, and $d$ to replace the worst-case direct link signal-tonoise ratio (SNR) at $\mathrm{D}$ and $\mathrm{E}_{k}$ for constraints $(18 \mathrm{~b})-(18 \mathrm{~d})$. First, we minimize the direct link SNR at D for constraint (18b), which is [26]

$$
a=\min _{\Delta h_{\mathrm{SD}} \in \mathcal{H}_{\mathrm{SD}}} P_{s}\left|h_{\mathrm{SD}}\right|^{2}=P_{s}|| \tilde{h}_{\mathrm{SD}}\left|-\delta_{\mathrm{SD}}\right|^{2} .
$$

Likewise, we maximize the direct link SNR at $\mathrm{E}_{k}$ for constraint (18c), which is

$$
b=\max _{\Delta h_{\mathrm{SE}_{k}} \in \mathcal{H}_{\mathrm{SE}_{k}}} P_{s}\left|h_{\mathrm{SE}_{k}}\right|^{2}=P_{s}|| \tilde{h}_{\mathrm{SE}_{k}}\left|+\delta_{\mathrm{SE}_{k}}\right|^{2}, \forall k \in \mathcal{K} .
$$

In addition, we maximize the direct link SNR at D for constraint (18d), which is

$$
c=\max _{\Delta h_{\mathrm{SD}} \in \mathcal{H}_{\mathrm{SD}}} P_{s}\left|h_{\mathrm{SD}}\right|^{2}=P_{s}|| \tilde{h}_{\mathrm{SD}}\left|+\delta_{\mathrm{SD}}\right|^{2} .
$$

For constraint (18b), since we have (19), it can be simplified as

$$
\min _{\Delta \mathbf{h}_{\mathrm{RD}} \in \mathcal{H}_{R D}} \frac{(1+a)+\mathbf{h}_{\mathrm{RD}}^{T}[\mathbf{W}+(1+a) \Omega] \mathbf{h}_{\mathrm{RD}}^{*}}{\alpha\left(1+\mathbf{h}_{\mathrm{RD}}^{T} \Omega \mathbf{h}_{\mathrm{RD}}^{*}\right)} \geq \gamma .
$$

Similarly, for constraints (18c) and (18d), since we have (20) and (21), then they can be respectively simplified as

$$
\begin{aligned}
& \max _{\Delta \mathbf{h}_{\mathrm{RE}_{k} \in \mathcal{H}}} \frac{(1+b)+\mathbf{h}_{\mathrm{RE}_{k}}^{T}[\mathbf{W}+(1+b) \Omega] \mathbf{h}_{\mathrm{RE}_{k}}^{*}}{1+\mathbf{h}_{\mathrm{RE}_{k}}^{T} \Omega \mathbf{h}_{\mathrm{RE}_{k}}^{*}} \\
& \leq \alpha, \forall k \in \mathcal{K}
\end{aligned}
$$

and

$$
\max _{\Delta \mathbf{h}_{\mathrm{RD}} \in \mathcal{H} \mathrm{RD}} c+\frac{\mathbf{h}_{\mathrm{RD}}^{T} \mathbf{W} \mathbf{h}_{\mathrm{RD}}^{*}}{1+\mathbf{h}_{\mathrm{RD}}^{T} \Omega \mathbf{h}_{\mathrm{RD}}^{*}} \leq d
$$

where $d=P_{s}\left\|\mathbf{h}_{\mathrm{SR}}\right\|^{2}$.

Combining (22)-(24), problem (17) is simplified as

$$
\max _{\mathbf{W}, \Omega, \alpha, \gamma} \gamma
$$

s.t. (22),(23),(24),

$$
\begin{aligned}
& \operatorname{tr}(\mathbf{W})+\operatorname{tr}(\Omega) \leq P_{r} \\
& \mathbf{W} \geq \mathbf{0}, \Omega \geq \mathbf{0}, \alpha \geq 0, \gamma \geq 0, \\
& \operatorname{rank}(\mathbf{W}) \leq 1
\end{aligned}
$$

To solve problem (25), we further introduce the following variables:

$$
t=\frac{1}{\alpha\left(1+\mathbf{h}_{\mathrm{RD}}^{H} \Omega \mathbf{h}_{\mathrm{RD}}\right)}, \hat{\mathbf{W}}=t \mathbf{W}, \text { and } \hat{\Omega}=t \Omega
$$

where $t \geq 0$ is a complimentary slackness parameter. Using Charnes-Cooper transformation [32], problem (25) is equivalently transformed as 


$$
\max _{\hat{\mathbf{W}}, \hat{\Sigma}, \gamma, \alpha, t} \gamma
$$

s.t. $\min _{\Delta \mathbf{h}_{\mathrm{RD}} \in \mathcal{H}_{\mathrm{RD}}}(1+a) t+\mathbf{h}_{\mathrm{RD}}^{T}[\hat{\mathbf{W}}+(1+a) \hat{\Omega}] \mathbf{h}_{\mathrm{RD}}^{*} \geq \gamma$,

$$
\begin{aligned}
& \max _{\Delta \mathbf{h}_{\mathrm{RE}_{k} \in \mathcal{H} \mathrm{RE}_{k}}} \frac{(1+b) t+\mathbf{h}_{\mathrm{RE}_{k}}^{T}[\hat{\mathbf{W}}+(1+b) \hat{\Omega}] \mathbf{h}_{\mathrm{RE}_{k}}^{*}}{t+\mathbf{h}_{\mathrm{RE}_{k}}^{T} \hat{\Omega} \mathbf{h}_{\mathrm{RE}_{k}}^{*}} \\
& \leq \alpha, \forall k \in \mathcal{K} \\
& \max _{\Delta \mathbf{h}_{\mathrm{RD}} \in \mathcal{H}_{\mathrm{RD}}} \alpha \mathbf{h}_{\mathrm{RD}}^{T} \hat{\mathbf{W}} \mathbf{h}_{\mathrm{RD}}^{*} \leq d-c, \\
& \alpha\left(t+\mathbf{h}_{\mathrm{RD}}^{T} \hat{\Omega} \mathbf{h}_{\mathrm{RD}}^{*}\right) \leq 1, \forall \Delta \mathbf{h}_{\mathrm{RD}} \in \mathcal{H}_{\mathrm{RD}}, \\
& \operatorname{tr}(\hat{\mathbf{W}})+\operatorname{tr}(\hat{\Omega}) \leq t P_{r}, \\
& \hat{\mathbf{W}} \succeq \mathbf{0}, \hat{\Omega} \succeq \mathbf{0}, \gamma \geq 0, \alpha \geq 0, t \geq 0, \\
& \operatorname{rank}(\hat{\mathbf{W}}) \leq 1 \text {. }
\end{aligned}
$$

Problem (27) has semi-infinite constraints (27b)-(27e) and non-convex rank-one constraint $(27 \mathrm{~h})$, which are intractable. Denoting the optimal solution to problem (27) as $\left(\hat{\mathbf{W}}^{o}, \hat{\Omega}^{o}, t^{o}\right)$ and the corresponding optimal objective value as $\gamma^{o}$, using SDR to obtain $\left(\hat{\mathbf{W}}^{o}, \hat{\Omega}^{o}, t^{o}\right)$ under given $\alpha$, and using 1-D line search to search the optimal $\alpha$, we have the following proposition.

Proposition 1. The optimal solution $\left(\hat{\mathbf{W}}^{o}, \hat{\Omega}^{o}, t^{o}\right)$ to problem (27) should satisfy the following equation:

$$
\max _{\Delta \mathbf{h}_{\mathrm{RD}} \in \mathcal{H}_{\mathrm{RD}}} \mathbf{h}_{\mathrm{RD}}^{T} \hat{\Omega}^{o} \mathbf{h}_{\mathrm{RD}}+t^{o}=1 / \alpha .
$$

Proof. Please refer to the "Appendix 1" section.

To make problem (27) tractable, we first employ the $S$-procedure [34] to convert the constraints (27b)-(27e) into linear matrix inequalities (LMIs) [35]. For constraint (27b), we substitute (13) into (27b) and rewrite it as follows

$$
\left\{\begin{array}{l}
-\Delta \mathbf{h}_{\mathrm{RD}}^{T} \Sigma_{1} \Delta \mathbf{h}_{\mathrm{RD}}-2 \operatorname{Re}\left\{\left(\tilde{\mathbf{h}}_{\mathrm{RD}} \Sigma_{1}\right)^{T} \Delta \mathbf{h}_{\mathrm{RD}}^{*}\right\}-\tilde{\mathbf{h}}_{\mathrm{RD}}^{T} \Sigma_{1} \tilde{\mathbf{h}}_{\mathrm{RD}}^{*}+\gamma-(1+a) t \leq 0 \\
\forall \Delta \mathbf{h}_{\mathrm{RD}}:\left\|\Delta \mathbf{h}_{\mathrm{RD}}\right\| \leq \delta_{R D}
\end{array}\right.
$$

where $\Sigma_{1}=\hat{\mathbf{W}}+(1+a) \hat{\Omega}$.

By applying $S$-procedure and introducing a slack variable $\lambda_{1} \geq 0$, we convert (29) into an LMI given by

$$
\mathbf{T}_{1}=\left[\begin{array}{cc}
\lambda_{1} \mathbf{I} / \delta_{\mathrm{RD}}^{2}+\Sigma_{1} & \Sigma_{1} \tilde{\mathbf{h}}_{\mathrm{RD}}^{*} \\
\tilde{\mathbf{h}}_{\mathrm{RD}}^{T} \Sigma_{1} & \tilde{\mathbf{h}}_{\mathrm{RD}}^{T} \Sigma_{1} \tilde{\mathbf{h}}_{\mathrm{RD}}^{*}+(1+a) t-\gamma-\lambda_{1}
\end{array}\right] \succeq \mathbf{0} .
$$

Similarly, the constrains in (27c)-(27e) are equivalently expressed as

$$
\begin{gathered}
\mathbf{T}_{2 k}=\left[\begin{array}{cc}
u_{k} \mathbf{I} / \delta_{\mathrm{RE}_{k}}^{2}+\Sigma_{2} & \Sigma_{2} \tilde{\mathbf{h}}_{\mathrm{RE}}^{*} \\
\tilde{\mathbf{h}}_{\mathrm{RE}}^{T} \Sigma_{2} & \tilde{\mathbf{h}}_{\mathrm{RE}}^{T} \Sigma_{2} \tilde{\mathbf{h}}_{\mathrm{RE}}^{*}+(\alpha-1-b) t-u_{k}
\end{array}\right] \succeq \mathbf{0}, \forall k \in \mathcal{K} \\
\mathbf{T}_{3}=\left[\begin{array}{cc}
\lambda_{2} \mathbf{I} / \delta_{\mathrm{RD}}^{2}-\Sigma_{\mathbf{3}} \\
-\tilde{\mathbf{h}}_{\mathrm{RD}}^{T} \Sigma_{\mathbf{3}} & -\tilde{\mathbf{h}}_{\mathrm{RD}}^{T} \Sigma_{\mathbf{3}} \tilde{\mathbf{h}}_{\mathrm{RD}}^{*}+d-c-\lambda_{2}
\end{array}\right] \succeq \mathbf{0} \\
\mathbf{T}_{4}=\left[\begin{array}{c}
\lambda_{3} \mathbf{I} / \delta_{\mathrm{RD}}^{2}-\hat{\Omega} \\
-\tilde{\mathbf{h}}_{\mathrm{RD}}^{T} \hat{\Omega}
\end{array}\right. \\
\left.\begin{array}{c}
\tilde{\mathbf{h}}_{\mathrm{RD}}^{H} \hat{\Omega} \tilde{\mathbf{h}}_{\mathrm{RD}}^{*}+1 / \alpha-t-\lambda_{3}
\end{array}\right] \succeq \mathbf{0}
\end{gathered}
$$

where $\Sigma_{2}=(\alpha-1-b) \hat{\Omega}-\hat{\mathbf{W}}$, and $\Sigma_{3}=\alpha \hat{\mathbf{W}} \cdot \lambda_{1} \geq 0$, $\lambda_{2} \geq 0, \lambda_{3} \geq 0$, and $u_{k} \geq 0$ are introduced slack variables.

Replacing (27b)-(27e) by (30)-(33), problem (27) can be equivalently expressed as

$$
\begin{aligned}
\underset{\hat{\mathbf{W}}, \hat{\Sigma}, \gamma, \alpha, t, \lambda_{1}, \lambda_{2}, \lambda_{3}, u_{k}}{\max } & \gamma \\
\text { s.t. } & (30),(31),(32),(33), \\
& \operatorname{tr}(\hat{\mathbf{W}})+\operatorname{tr}(\hat{\Omega}) \leq t P_{r}, \\
& \hat{\mathbf{W}} \geq \mathbf{0}, \hat{\Omega} \geq \mathbf{0}, \\
& \lambda_{1} \geq 0, \lambda_{2} \geq 0, \lambda_{3} \geq 0, u_{k} \geq 0, \forall k \in \mathcal{K} \\
& \gamma \geq 0, \alpha \geq 0, t \geq 0, \\
& \operatorname{rank}(\hat{\mathbf{W}}) \leq 1 .
\end{aligned}
$$

Problem (34) is still non-convex due to the rank-one constraint of $\hat{\mathbf{W}}$. To meet this challenge, we use the SDR technique here. By dropping the rank-one constraint, problem (34) becomes a convex semidefinite program (SDP) for a given $\alpha$ that consists of a linear objective function together with a set of LMI constraints, which can thus be solved effectively via standard convex optimization techniques such as the interior point method [35]. As a result, we can recast problem (34) as a two-layer optimization problem.

The outer layer is a one-variable optimization problem in terms of $\alpha$, and the inner layer is a convex SDP problem with fixed $\alpha$. Thus, we need to search for $\alpha$. The upper bound of $\alpha$ is derived as

$$
\begin{aligned}
\alpha \leq 1+\frac{\mathbf{h}_{\mathrm{RD}}^{T} \mathbf{W} \mathbf{h}_{\mathrm{RD}}^{*}}{1+\mathbf{h}_{\mathrm{RD}}^{T} \Omega \mathbf{h}_{\mathrm{RD}}^{*}} & \leq 1+P_{r}\left\|\mathbf{h}_{\mathrm{RD}}\right\|^{2} \\
& =1+P_{r}\left(\left\|\tilde{\mathbf{h}}_{\mathrm{RD}}\right\|+\delta_{\mathrm{RD}}\right)^{2} .
\end{aligned}
$$


Toward this end, we reformulate problem (34) in the following form

$$
\begin{aligned}
& \max _{\alpha} f(\alpha) \\
& \text { s.t. } \quad 1 \leq \alpha \leq 1+P_{r}\left(\left\|\tilde{\mathbf{h}}_{\mathrm{RD}}\right\|+\delta_{\mathrm{RD}}\right)^{2}
\end{aligned}
$$

where

$$
f(\alpha)=\max _{\hat{\mathbf{W}}, \hat{\Sigma}, \gamma, \alpha, t, \lambda_{1}, \lambda_{2}, \lambda_{3}, u_{k}}
$$

s.t. all constraints in problem (34).

Here, if $\hat{\mathbf{W}}^{o}$ is of rank-one, then the solution of the SDR is the optimal solution to the original problem (34) (in other words, the SDR of problem (34) is tight). In this case, the optimal robust beamforming vector at $\mathrm{R}$ can be exactly obtained as $\mathbf{w}^{o}$ via employing the eigenvalue decomposition $\mathbf{W}^{o}=\mathbf{w}^{o}\left(\mathbf{w}^{o}\right)^{H}$; otherwise, it is not in general. Fortunately, due to the specific structure of problem (34), we can prove in the following that $\hat{\mathbf{W}}^{o}$ is indeed of rank one. Accordingly, the obtained optimal solution to the SDR of (34) is indeed the optimal solution to the original problem (34). The robust beamforming design without direct transmission (DT) from S to D and from $\mathrm{S}$ to Es is a special case of (34) with $a=0, b=0$.

Proposition 2. The optimal solution to the SDR of problem (34), $\hat{\mathbf{W}}^{o}$, is always of rank one.

Proof. Please refer to the "Appendix 2" section.
Remark 1. It is obvious that the main computation complexity stems from the computation of $\hat{\mathbf{W}}^{o}$ in the inner layer. The complexity of solving an SDP problem is $\mathcal{O}\left(n_{\text {sdp }}^{0.5}\left(m_{\text {sdp }} n_{\text {sdp }}^{3}+m_{\text {sdp }}^{2} n_{\text {sdp }}^{2}+m_{\text {sdp }}^{3}\right)\right) \log (1 / \epsilon)$ according to [36]. Here, $m_{\text {sdp }}$ denotes the number of semidefinite cone constraints, $n_{\text {sdp }}$ denotes the dimension of the semidefinite cone, and $\epsilon$ is the accuracy of solving the SDP. Comparing the SDP of (34) with the standard form in [36], we have $m_{\mathrm{sdp}}=K+4$ and $n_{\mathrm{sdp}}=N+1$. As a result, the computational complexity of the proposed scheme is

$$
\begin{aligned}
& \mathcal{O}\left\{L \left[(K+4)(N+1)^{3.5}+(K+4)^{2}(N+1)^{2.5}\right.\right. \\
& \left.\left.+(K+4)^{3}(N+1)^{0.5}\right]\right\} \log (1 / \epsilon)
\end{aligned}
$$

where $L$ is the number of 1-D search.

\section{Numerical results}

In this section, we present simulation results to show the performance of the proposed robust joint beamforming and CJ design in DF relay system under channel uncertainty. We assume relay has four antennas, i.e., $N=4$, and the number of eavesdroppers is $K=3$. All the channel coefficients are assumed to be independent and identically distributed (i.i.d.) CSCG random variables with zero mean and unit variance. The transmit power at $\mathrm{S}$ is $P_{s}=0 d B$. For simplicity, we assume the error bounds are the same, i.e., $\delta_{\mathrm{SD}}=\delta_{\mathrm{SE}_{k}}=\delta_{\mathrm{RD}}=\delta_{\mathrm{RE}_{k}}=\delta$. We average the worstcase secrecy rate via conducting 1000 randomly generated channel realizations.

Figure 2 presents the average secrecy rate comparison of our proposed robust beamforming with $\mathrm{CJ}$ scheme

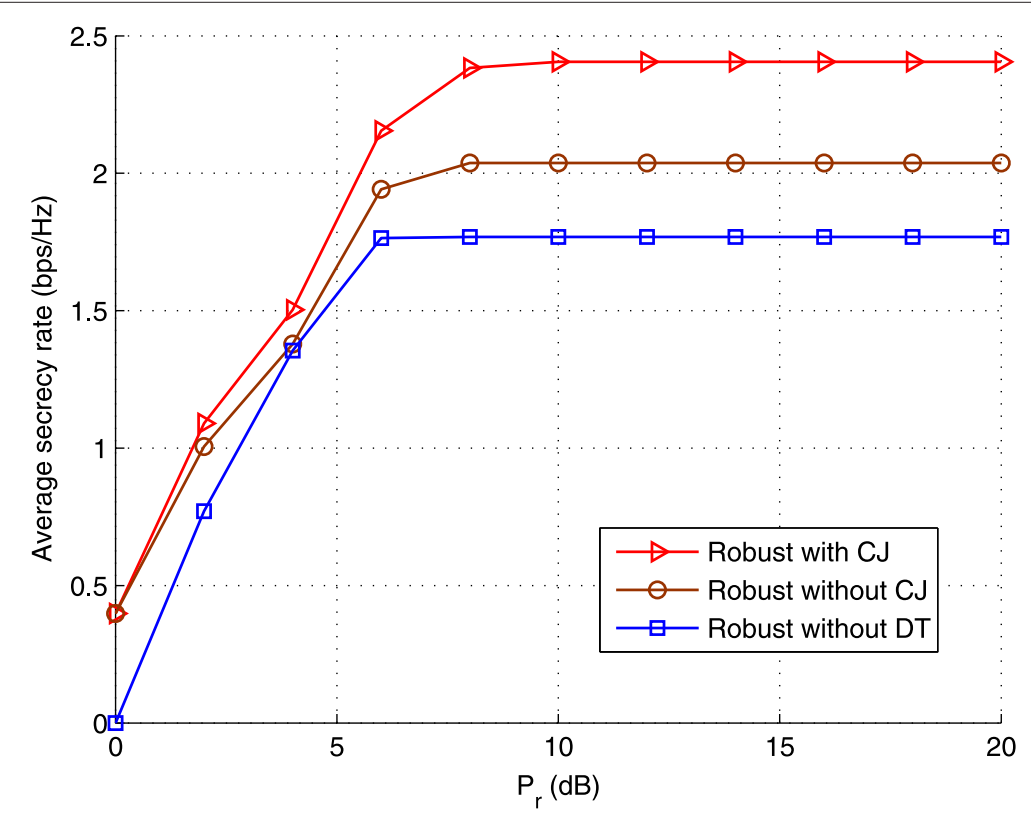

Fig. 2 Average secrecy rate versus the maximum total transmit power constraint $P_{r}$ at $\mathrm{R}$ under the error bound $\delta=0.2$ 


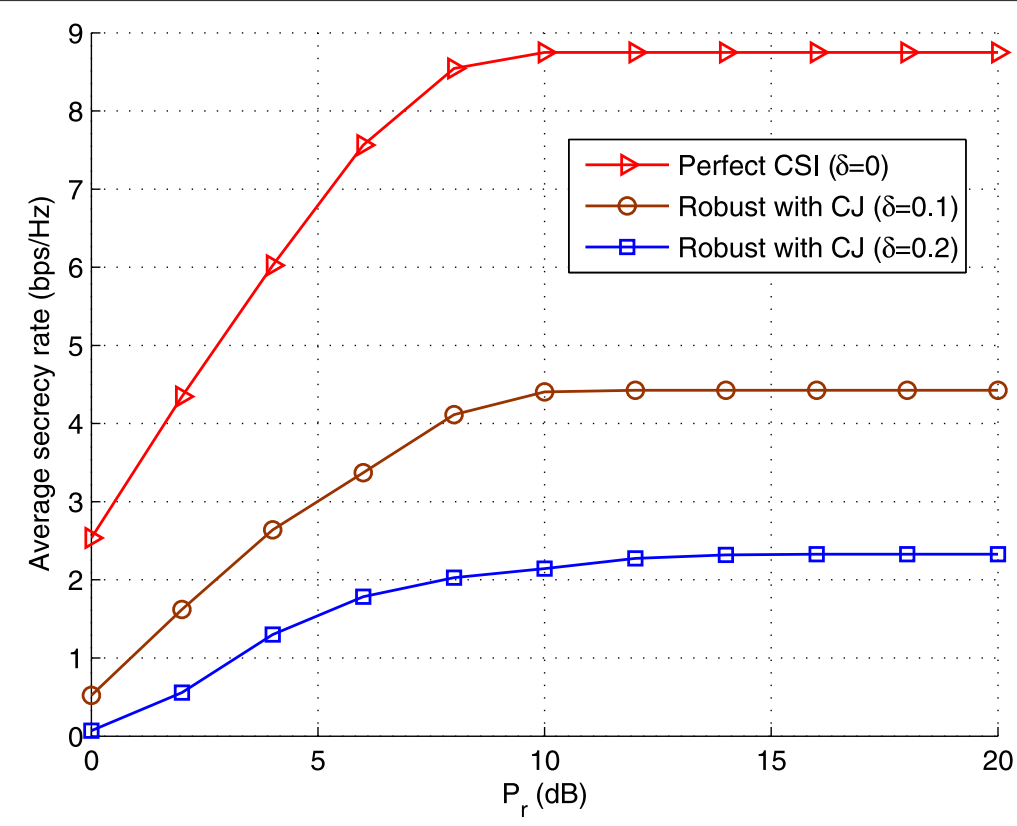

Fig. 3 Average secrecy rate versus the maximum total transmit power constraint $P_{r}$ at R for different error bound $\delta$

(denoted as "robust with CJ"), robust beamforming without CJ scheme (denoted as "robust without CJ"), and robust beamforming without DT scheme (denoted as "robust without DT") under the error bound $\delta=0.2$. Here, the robust without CJ scheme is obtained by solving problem (34) with $\Omega=0$ [26]. From Fig. 2, it is observed that the average secrecy rate increases as the maximum total transmit power of relay increases and our proposed robust beamforming with $\mathrm{CJ}$ scheme is superior to all its counterparts in improving the secrecy rate.

Figure 3 shows the average secrecy rate versus the maximum total transmit power constraint at $\mathrm{R}$ for the comparison of both imperfect CSI and perfect CSI cases. It is observed that the average secrecy rate for perfect CSI is higher than that in imperfect CSI cases. As the

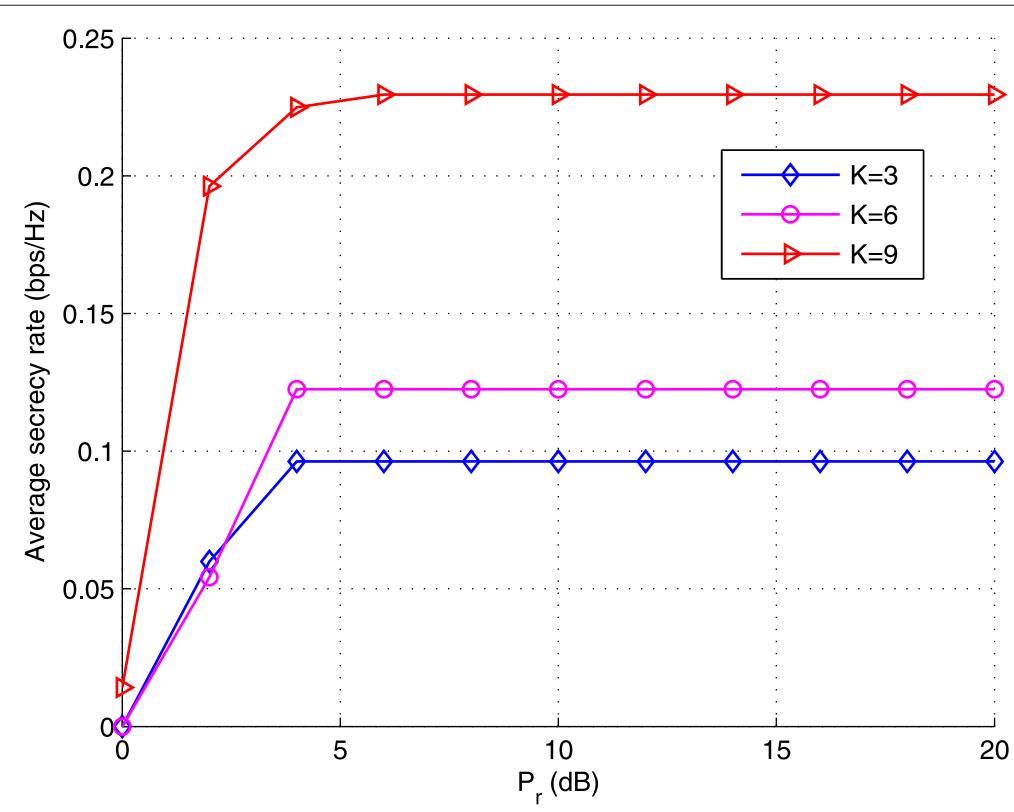

Fig. 4 Comparison between different number of eavesdroppers for fixed $\delta=0.2$, i.e., $K=3, K=6$ and $K=9$, respectively 


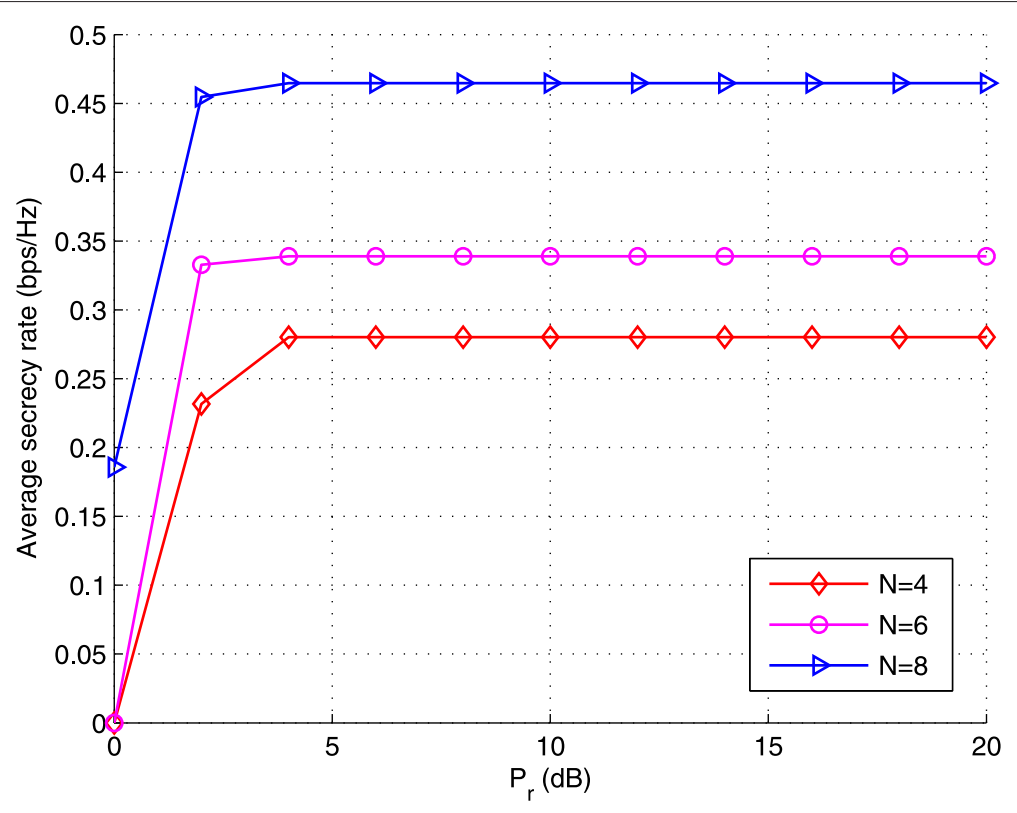

Fig. 5 Comparison between different transmit antennas at relay for fixed $\delta=0.2$, i.e., $N=4, N=6$ and $N=8$, respectively

uncertainty bounds increase, the system becomes more roust against uncertainty; however, the achievable secrecy rate degrades. From Fig. 3, it is also observed that the secrecy rate increases with increase of maximum total transmit power at $\mathrm{R}$ and exhibits saturation behavior in high relay transmit power due to the constraint (10).

Figure 4 plots the average secrecy rate for $K=3,6,9$ eavesdroppers under $N=4$, respectively. It can be observed that as eavesdropper number $K$ increases, the average secrecy rate would decrease. More eavesdroppers would surely degrade system performance. Figure 5 depicts the comparison of the average secrecy rate for different transmit antennas at relay. It is shown in Fig. 5 that the average secrecy rate is lifted to a higher level when more transmit antennas are equipped on relay. This is due to the fact that when the number of antennas increases, it means that there are more channels in the relay system and then more spatial degrees of freedom are utilized. Since the secrecy rate of each channel is non-negative, the increasing number of channels means an increase of system secrecy rate.

\section{Conclusions}

In this paper, we investigated robust beamforming and CJ design for a DF-based relay system in the presence of multiple eavesdroppers. We jointly optimized the transmit beamforming vector and the covariance matrix of jamming signals at the relay to maximize the achievable secrecy rate at the destination while ensuring the total transmit power constraint at the relay, in which we took into account imperfect CSI subject to norm-bounded errors. By applying the techniques of SDR together with a 1-D search, we have obtained the optimal solution. Furthermore, we proved that the SDR of the problem is tight. Simulation results have shown that the validity of the proposed scheme.

\section{Appendix 1}

Proof of proposition 1

If $\left(\hat{\mathbf{W}}^{o}, \hat{\Omega}^{o}, t^{o}\right)$ is optimal solution of problem (27), we have

$$
\min _{\Delta \mathbf{h}_{\mathrm{RD}} \in \mathcal{H}_{\mathrm{RD}}}(1+a) t+\mathbf{h}_{\mathrm{RD}}^{T}\left[\hat{\mathbf{W}}^{o}+(1+a) \hat{\Omega}^{o}\right] \mathbf{h}_{\mathrm{RD}}^{*}=\gamma^{o}
$$

Following the idea of [33] to prove this proposition, by reduction ad absurdum, we consider the following two situations. If $\max _{\Delta \mathbf{h}_{\mathrm{RD}} \in \mathcal{H}_{\mathrm{RD}}} \mathbf{h}_{\mathrm{RD}}^{T} \hat{\Omega}^{o} \mathbf{h}_{\mathrm{RD}}^{*}+t^{o} \leq 1 / \alpha$ and $\operatorname{tr}\left(\hat{\mathbf{W}}^{o}\right)+\operatorname{tr}\left(\hat{\Omega}^{o}\right)<t^{o} P_{r}$, we can always find $t=t^{o}+$ $\Delta t, \Delta t>0$, which satisfies constraints (27c)-(27f). Thus, we can find the large objective value $\gamma^{o}+\Delta t$ which satisfies (39). If $\max _{\Delta \mathbf{h}_{\mathrm{RD}} \in \mathcal{H}_{\mathrm{RD}}} \mathbf{h}_{\mathrm{RD}}^{T} \hat{\Omega}^{o} \mathbf{h}_{\mathrm{RD}}^{*}+t^{o} \leq 1 / \alpha$ and $\operatorname{tr}\left(\hat{\mathbf{W}}^{o}\right)+\operatorname{tr}\left(\hat{\Omega}^{o}\right)=t^{o} P_{r}$, we can find a solution $\left(\hat{\mathbf{W}}^{o}, \hat{\Omega}^{o}+\Delta \hat{\Omega}, t^{o}+\Delta t\right), \Delta \hat{\Omega} \succ \mathbf{0}, \Delta t>0$, which makes the constraint $\max _{\Delta \mathbf{h}_{\mathrm{RD}} \in \mathcal{H}_{\mathrm{RD}}} \mathbf{h}_{\mathrm{RD}}^{T} \hat{\Omega}^{o} \mathbf{h}_{\mathrm{RD}}^{*}+t^{o} \leq 1 / \alpha$ tighter while satisfying constraints $(27 \mathrm{c}),(27 \mathrm{~d})$. As a result, these contradict that $\left(\hat{\mathbf{W}}^{o}, \hat{\Omega}^{o}, t^{o}\right)$ is the optimal solution to problem (27).

\section{Appendix 2}

\section{Proof of proposition 2}

We prove the rank condition of $\hat{\mathbf{W}}$ through KarushKuhn-Tucker (KKT) conditions. The partial Lagrangian 
of primal problem (34) with respect to $\hat{\mathbf{W}}$ is given by

$$
\begin{aligned}
\mathcal{L}= & \gamma-\operatorname{tr}\left(\mathbf{B}_{1} \mathbf{T}_{1}\right)-\operatorname{tr}\left(\mathbf{B}_{2 k} \mathbf{T}_{2 k}\right)-\operatorname{tr}\left(\mathbf{B}_{3} \mathbf{T}_{3}\right) \\
& +\mu\left[\operatorname{tr}(\hat{\mathbf{W}})+\operatorname{tr}(\hat{\Omega})-t P_{r}\right]-\operatorname{tr}\left(\mathbf{B}_{4} \hat{\mathbf{W}}\right)
\end{aligned}
$$

where the dual variables $\mathbf{B}_{1} \in \mathbb{H}_{+}, \mathbf{B}_{2 k} \in \mathbb{H}_{+}, \mathbf{B}_{3} \in \mathbb{H}_{+}$, $\mathbf{B}_{4} \in \mathbb{H}_{+}\left(\mathbb{H}_{+}\right.$denotes Hermitian matrix $)$, and $\mu \geq 0$ are corresponding to the constraints $\mathbf{T}_{1} \succeq \mathbf{0}, \mathbf{T}_{2 k} \succeq \mathbf{0}, \mathbf{T}_{3} \succeq \mathbf{0}$, $\hat{\mathbf{W}} \succeq \mathbf{0}$, and $\operatorname{tr}(\hat{\mathbf{W}})+\operatorname{tr}(\hat{\Omega})-t P_{r} \leq 0$ in (34), respectively.

Taking the partial derivative of (40) with respect to $\hat{\mathbf{W}}$ and applying KKT conditions, we have

$$
\begin{aligned}
& \mu \mathbf{I}-\mathbf{H}_{\mathrm{RD}} \mathbf{B}_{1} \mathbf{H}_{\mathrm{RD}}^{H}+\sum_{k=1}^{K} \mathbf{H}_{\mathrm{RE}_{k}} \mathbf{B}_{2 k} \mathbf{H}_{\mathrm{RE}_{k}}^{H}+\mathbf{H}_{\mathrm{RD}} \mathbf{B}_{3} \mathbf{H}_{\mathrm{RD}}^{H} \\
& -\mathbf{B}_{4}=\mathbf{0}
\end{aligned}
$$

where $\mathbf{H}_{\mathrm{RD}}=\left[\mathbf{I}_{N}, \tilde{\mathbf{h}}_{\mathrm{RD}}\right], \mathbf{H}_{\mathrm{RE}_{k}}=\left[\mathbf{I}_{N}, \tilde{\mathbf{h}}_{\mathrm{RE}_{k}}\right]$. It is noted that $\mathbf{B}_{4} \hat{\mathbf{W}}=\mathbf{0}$ from KKT conditions. Since the size of $\mathbf{B}_{1}$ and $\mathbf{T}_{1}$ is $(N+1) \times(N+1)$, we have $\operatorname{rank}\left(\mathbf{B}_{1}\right)+\operatorname{rank}\left(\mathbf{T}_{1}\right) \leq$ $N+1$. Since the dimension of $\mathbf{B}_{1}$ and $\mathbf{T}_{1}$ is $(N+1) \times(N+1)$, according to the Sylvester's rank inequality [37], we have $\operatorname{rank}\left(\mathbf{B}_{1}\right)+\operatorname{rank}\left(\mathbf{T}_{1}\right)-(N+1) \leq \operatorname{rank}\left(\mathbf{B}_{1} \mathbf{T}_{1}\right)$, then $\operatorname{rank}\left(\mathbf{B}_{1}\right)+\operatorname{rank}\left(\mathbf{T}_{1}\right) \leq N+1$.

From $S$-procedure [34], we get the slack variable $\lambda_{1} \geq 0$. Next, we show that $\lambda_{1}>0$ via reduction ad absurdum. We consider the following situation. If $\lambda_{1}=0$, the constraint $\left\|\Delta \mathbf{h}_{\mathrm{RD}}\right\| \leq \delta_{\mathrm{RD}}$ is not active because $\lambda_{1}$ is its dual variable associated with the constraint in (30). If $\left\|\Delta \mathbf{h}_{\mathrm{RD}}^{\star}\right\| \leq$ $\delta_{\mathrm{RD}},\left\|\Delta \mathbf{h}_{\mathrm{RD}}^{\star}\right\|$ is the worst channel uncertainty to minimize $(1+a) t+\mathbf{h}_{\mathrm{RD}}^{T}[\hat{\mathbf{W}}+(1+a) \hat{\Omega}] \mathbf{h}_{\mathrm{RD}}^{*}$ in constraint (27b), we can always find a scaler $\rho>1$ which satisfies $\left\|\rho \Delta \mathbf{h}_{\mathrm{RD}}^{\star}\right\| \leq \delta_{\mathrm{RD}}$. By substituting $\rho \Delta \mathbf{h}_{\mathrm{RD}}^{\star}$ into $(1+a) t+$ $\mathbf{h}_{\mathrm{RD}}^{T}[\hat{\mathbf{W}}+(1+a) \hat{\Omega}] \mathbf{h}_{\mathrm{RD}}^{*}$, we get the lower value than that obtained by $\Delta \mathbf{h}_{\mathrm{RD}}^{\star}$. It is contradictory to the assumption that $\Delta \mathbf{h}_{\mathrm{RD}}^{\star}$ minimizes $(1+a) t+\mathbf{h}_{\mathrm{RD}}^{T}[\hat{\mathbf{W}}+(1+a) \hat{\Omega}] \mathbf{h}_{\mathrm{RD}}^{*}$. As a result, $\operatorname{rank}\left(\mathbf{T}_{1}\right) \geq N$ and $\operatorname{rank}\left(\mathbf{B}_{1}\right) \leq 1$ hold when $\lambda_{1}>$ 0 . Furthermore, $\operatorname{rank}\left(\mathbf{B}_{1}\right) \neq 0$, so we have $\operatorname{rank}\left(\mathbf{B}_{1}\right)=1$. Similarly, we have $\operatorname{rank}\left(\mathbf{B}_{2 k}\right)=\operatorname{rank}\left(\mathbf{B}_{3}\right)=\operatorname{rank}\left(\mathbf{B}_{4}\right)=1$. Multiplying both sides of (41) with $\hat{\mathbf{W}}$

$$
\begin{aligned}
\left(\mu \mathbf{I}+\mathbf{H}_{\mathrm{RE}_{k}} \mathbf{B}_{2} \mathbf{H}_{\mathrm{RE}}^{H}+\mathbf{H}_{\mathrm{RD}} \mathbf{B}_{3} \mathbf{H}_{\mathrm{RD}}^{H}\right) \hat{\mathbf{W}} & =\left(\mathbf{B}_{4}+\mathbf{H}_{\mathrm{RD}} \mathbf{B}_{1} \mathbf{H}_{\mathrm{RD}}^{H}\right) \hat{\mathbf{W}} \\
& =\mathbf{H}_{\mathrm{RD}} \mathbf{B}_{1} \mathbf{H}_{\mathrm{RD}}^{H} \hat{\mathbf{W}}
\end{aligned}
$$

Since $\mu \mathbf{I}+\mathbf{H}_{\mathrm{RE}_{k}} \mathbf{B}_{2} \mathbf{H}_{\mathrm{RE}_{k}}^{H}+\mathbf{H}_{\mathrm{RD}} \mathbf{B}_{3} \mathbf{H}_{\mathrm{RD}}^{H} \succ \mathbf{0}$ has full rank, we have

$$
\operatorname{rank}(\hat{\mathbf{W}})=\operatorname{rank}\left(\mathbf{H}_{\mathrm{RD}} \mathbf{B}_{1} \mathbf{H}_{\mathrm{RD}}^{H} \hat{\mathbf{W}}\right)
$$

Due to the fact that $\operatorname{rank}\left(\mathbf{B}_{1}\right)=1$ from the above proof, we obtain rank $\left(\mathbf{H}_{\mathrm{RD}} \mathbf{B}_{1} \mathbf{H}_{\mathrm{RD}}^{H}\right)=1$; thus, we have $\operatorname{rank}(\hat{\mathbf{W}}) \leq 1$. As $R_{D}(\hat{\mathbf{W}}, \hat{\Omega})>0, \operatorname{rank}(\hat{\mathbf{W}})$ must not be 0 , we have $\operatorname{rank}(\hat{\mathbf{W}})=1$. This completes the proof.
Competing interests

The authors declare that they have no competing interests.

\section{Acknowledgements}

This work was supported by the National Natural Science Foundation of China (Grant No. 61371075 and 61421001) and 863 Project (Grant No. 2015AA01A708).

Received: 5 August 2015 Accepted: 15 February 2016

Published online: 01 March 2016

\section{References}

1. Y Liang, HV Poor, S Shamai, Secure communication over fading channels. IEEE Trans. Inf. Theory. 54(6), 2470-2492 (2008)

2. M Dehghan, DL Goeckel, M Ghaderi, Z Ding, Energy efficiency of cooperative jamming strategies in secure wireless networks. IEEE Trans. Wirel. Commun. 11(9), 3025-3029 (2012)

3. Q Li, M Hong, HT Wai, YF Liu, WK Ma, ZQ Luo, Transmit solutions for MIMO wiretap channels using alternating optimization. IEEE J. Sel. Areas Commun. 31(9), 1714-1727 (2013)

4. AP Shrestha, KS Kwak, Performance of opportunistic scheduling for physical layer security with transmit antenna selection. EURASIP J. Wirel. Commun. Netw. 1(33), 1-9 (2014)

5. Y Zou, J Zhu, X Wang, VCM Leung. Improving physical-layer security in wireless communications using diversity techniques.IEEE Netw. 29(1), 42-48 (2015)

6. CWang, HM Wang, Robust joint beamforming and jamming for secure AF networks: low-complexity design. IEEE Trans. Veh. Technol. 64(5), 2192-2198 (2014)

7. TM Hoang, TQ Duong, HA Suraweera, C Tellambura, HV Poor, Cooperative beamforming and user selection for improving the security of relay-aided systems. IEEE Trans. Commun. 63(12), 5039-5051 (2015)

8. SAA Fakoorian, AL Swindlehurst, Solutions for the MIMO Gaussian wiretap channel with a cooperative jammer. IEEE Trans. Signal Process. 59(10), 5013-5022 (2011)

9. J Li, AP Petropulu, S Weber, On cooperative relaying schemes for wireless physical layer security. IEEE Trans. Signal Process. 59(10), 4985-4997 (2011)

10. M Ghaderi, DL Goeckel, A Orda, M Dehghan, in Proceedings of 10th Annual IEEE Communications Society Conference on Sensor, Mesh and Ad Hoc Communications and Networks (SECON). Efficient Wireless Security Through Jamming, Coding and Routing, (New Orleans, LA, 2013), pp. 505-513

11. M Ghaderi, D Goeckel, A Orda, M Dehghan, Minimum energy routing and jamming to thwart wireless network eavesdroppers. IEEE Trans. Mobile Comput. 14(7), 1433-1448 (2014)

12. L Dong, Z Han, AP Petropulu, HV Poor, Improving wireless physical layer security via cooperating relays. IEEE Trans. Signal Process. 58(3), 1875-1888 (2010)

13. G Zheng, LC Choo, KK Wong, Optimal cooperative jamming to enhance physical layer security using relays. IEEE Trans. Signal Process. 59(3), 1317-1322 (2011)

14. PH Lin, SH Lai, SC Lin, HJ Su, On secrecy rate of the generalized artificial-noise assisted secure beamforming for wiretap channels. IEEE J. Sel. Areas Commun. 31(9), 1728-1740 (2013)

15. Q Li, WK Ma, Spatially selective artificial-noise aided transmit optimization for MISO multi-eves secrecy rate maximization. IEEE Trans. Signal Process. 61(10), 2704-2717 (2013)

16. Z Chu, K Cumanan, Z Ding, M Johnston, S Le Goff, Secrecy rate optimizations for a MIMO secrecy channel with a cooperative jammer. IEEE Trans. Veh. Technol. 64(5), 1833-1847 (2015)

17. Y Yang, Q Li, WK Ma, J Ge, PC Ching, Cooperative secure beamforming for AF relay networks with multiple eavesdroppers. IEEE Signal Process. Lett. 20(1), 35-38 (2013)

18. Z Ding, M Peng, $\mathrm{HH}$ Chen, A general relaying transmission protocol for MIMO secrecy communications. IEEE Trans. Commun. 60(11), 3461-3471 (2012)

19. S Vishwakarma, A Chockalingam, in Twentieth National Conference on Communications (NCC). MIMO Decode-and-Forward Relay Beamforming for Secrecy with Cooperative Jamming (IEEE, Kanpur, 2014), pp. 1-6 
20. X Wang, K Wang, X Zhang, Secure relay beamforming with imperfect channel state information. IEEE Trans. Veh. Technol. 62(5), 2140-2155 (2013)

21. J Huang, AL Swindlehurst, Robust secure transmission in MISO channels based on worst-case optimization. IEEE Trans. Signal Process. 60(4), 1696-1707 (2012)

22. $\mathrm{P}$ Zhao, M Zhang, H Yu, H Luo, W Chen, Robust beamforming design for sum secrecy rate optimization in MU-MISO networks. IEEE Trans. Inf. Forensics Secur. 10(9), 1812-1823 (2015)

23. J Ni, Z Fei, KK Wong, C Xing, J Kuang, K-F Tong, Robust coordinated beamforming for secure MISO interference channels with bounded ellipsoidal uncertainties. IEEE Wirel. Commun. Lett. 2(4), 407-410 (2013)

24. Z Fei, J Ni, D Zhao, C Xing, N Wang, J Kuang, Ergodic secrecy rate of two-user MISO interference channels with statistical CSI.SCIENCE CHINA Inf. Sci. 57(10), 1-14 (2014)

25. S Vishwakarma, A Chockalingam, in Proceedings of IEEE International Conference on Communications (ICC). Amplify-and-Forward Relay Beamforming for Secrecy with Cooperative Jamming and Imperfect CSI, (Budapest, 2013), pp. 3047-3052

26. M Zhang, H Yi, H Yu, H Luo, W Chen, Joint optimization in bidirectional multi-user multi-relay MIMO systems: non-robust and robust cases. IEEE Trans.Veh. Technol. 62(7), 3228-3244 (2013)

27. Y Qin, M Ding, M Zhang, $H$ Yu, H Luo, Relaying robust beamforming for device-to-device communication with channel uncertainty. IEEE Commun.Lett. 18(10), 1859-1862 (2014)

28. C Liu, N Yang, J Yuan, R Malaney, Location-based secure transmission for wiretap channels. IEEE J. Sel. Areas Commun. 33(7), 1458-1470 (2015)

29. F He, H Man, W Wang, Maximal ratio diversity combining enhanced security. IEEE Commun. Lett. 15(5), 509-511 (2011)

30. MZI Sarkar, T Ratnarajah. Enhancing security in correlated channel with maximal ratio combining diversity.IEEE Trans. on Signal Process. 60(12), 6745-6751 (2012)

31. M Tian, X Huang, Q Zhang, J Qin, Robust AN-aided secure transmission scheme in MISO channels with simultaneous wireless information and power transfer. IEEE Signal Process. Lett. 22(6), 723-727 (2015)

32. ZQ Luo, JF Sturm, S Zhang, Multivariate nonnegative quadratic mappings.SIAM. J. Optim. 14(4), 1140-1162 (2004)

33. R Feng, Q Li, Q Zhang, J Qin, Robust secure transmission in MISO simultaneous wireless information and power transfer system. IEEE Trans. Veh. Technol. 60(1), 400-405 (2015)

34. A Beck, YC Eldar, Strong duality in nonconvex quadratic optimization with two quadratic constraints.SIAM. J. Optim. 17(3), 844-860 (2006)

35. S Boyd, L Vandenberghe, Convex Optimization. (Cambridge University Press, Cambridge, 2004)

36. I Polik, T Terlaky, in Nonlinear Optimization, ed. by G Di Pillo, F Schoen, and 1. Interior Point Methods for Nonlinear Optimization (Springer, Berlin, 2010). ch.4

37. RA Horn, CR Johnson, Matrix Analysis. (Cambridge University Press, Cambridge, 1985)

\section{Submit your manuscript to a SpringerOpen ${ }^{\circ}$ journal and benefit from:}

- Convenient online submission

Rigorous peer review

- Immediate publication on acceptance

- Open access: articles freely available online

- High visibility within the field

- Retaining the copyright to your article

Submit your next manuscript at springeropen.com 